\title{
The soluble CD40 ligand sCD154 in systemic lupus erythematosus
}

\author{
Kazunori Kato, ${ }^{1}$ Ernesto Santana-Sahagún, ${ }^{2}$ Laura Z. Rassenti, ${ }^{1}$ Michael H. Weisman, ${ }^{2}$ \\ Naoto Tamura, ${ }^{3}$ Shigeto Kobayashi, ${ }^{3}$ Hiroshi Hashimoto, ${ }^{3}$ and Thomas J. Kipps ${ }^{1}$ \\ ${ }^{1}$ Division of Hematology/Oncology, and \\ ${ }^{2}$ Division of Rheumatology, University of California-San Diego School of Medicine, La Jolla, California 92093, USA \\ ${ }^{3}$ Department of Rheumatology, Juntendo University School of Medicine, Tokyo 113, Japan \\ Address correspondence to: Thomas J. Kipps, Division of Hematology/Oncology, \\ University of California-San Diego School of Medicine, 9500 Gilman Drive, La Jolla, California 92093-0663, USA. \\ Phone: (619) 534-5417; E-mail: tkipps@ucsd.edu. \\ Received for publication February 2, 1999, and accepted in revised form August 17, 1999.
}

\begin{abstract}
We found that the plasma of patients with active systemic lupus erythematosus (SLE) could induce a human B-cell line (Ramos) to express high levels of immune accessory molecules that are commonly found on blood B cells of patients with active SLE. The ability of SLE plasma to induce such phenotypic changes could be abrogated by neutralizing antibodies specific for the CD40 ligand (CD154) but not by antibodies to TNF- $\alpha$. Immunoprecipitation studies with anti-CD154 identified a 20-kDa protein in the plasma of SLE patients with active disease, but not in plasma of normal donors, indicating that such plasma contained soluble CD154 (sCD154). Using a quantitative ELISA method, we found that the plasma of patients with active disease had levels of sCD154 that were significantly higher than those found in plasma of normal donors. Levels of CD154 transcripts in SLE blood lymphocytes correlated with the relative concentrations of sCD154 found in SLE plasma. Furthermore, plasma levels of sCD154 correlated with the titers of anti-double-stranded DNA autoantibody and with clinical disease activity. These studies indicate that SCD154 of patients with SLE may act as a functional ligand for CD40 that is associated with SLE disease activity.
\end{abstract}

J. Clin. Invest. 104:947-955 (1999).

\section{Introduction}

Systemic lupus erythematosus (SLE) is an autoimmune disease characterized by prominent lymphocyte activation, resulting in production of pathogenic IgG autoantibodies such as anti-double-stranded DNA antibody (anti-dsDNA Ab). Such autoantibodies may play a critical role in the progression of lupus nephritis (1). Whereas low amounts of low-affinity anti-dsDNA $\mathrm{Ab}$ also can be detected in the sera of healthy adults, high serum titers of anti-dsDNA Ab's of the IgG isotype are found nearly exclusively in patients with SLE. Moreover, the levels of such IgG anti-dsDNA Ab's appear related to disease activity.

Aberrant expression of immune costimulatory molecules may contribute to this pathophysiology. Studies indicate that the blood lymphocytes of SLE patients often express higher levels of immune accessory molecules, such as CD54, CD80, CD86, and CD95, than the blood lymphocytes of normal adults (2-5). High-level expression of CD80 or CD86 may contribute to pathologic presentation of self antigens to $T$ cells and/or the production of pathologic antiDNA autoantibodies. Consistent with this notion, the production of pathologic autoantibodies by lupusprone New Zealand black (NZB) and New Zealand white (NZW) $\mathrm{F}_{1}$ mice can be ameliorated by CTLA4-Ig $(6,7)$, a recombinant protein that can block
CD80/CD86 $\leftrightarrow$ CD28 interactions (8). This has led to speculation that aberrant expression of these costimulatory molecules may contribute to the T-cell activation seen in patients with this disease.

Normal B cells can be induced to express immune costimulatory molecules by activated T cells. Activated CD4 T cells can express CD40 ligand (CD154), a molecule that can engage CD40 on the B-cell surface (9). This triggers a cascade of events that ultimately results in expression of a variety of heretofore nonexpressed stimulatory surface accessory molecules, such as CD80 (B7-1) (10-15). High-level expression of CD154 has also been detected on $T$ cells from patients with active SLE, indicating that such cells may have exaggerated expression of this stimulatory molecule $(16,17)$.

Conceivably, the exaggerated expression of CD154 could account for the high-level expression of immune accessory molecules on $B$ cells of patients with active disease. Moreover, high-level expression of CD154 may be required for disease activity, which appears to be the case in animal models of SLE. Early et al., for example, reported that the anti-dsDNA $\mathrm{Ab}$ production in $\mathrm{NZB} / \mathrm{NZW} \mathrm{F}_{1}$ mice could be suppressed by treatment with anti-mouse CD154 antibody in vivo (18). Also, lpr/lpr mice made genetically defective in their ability to express CD154 did not develop IgG rheumatoid factor or anti-dsDNA (19). 
On the other hand, soluble proteins released from activated $T$ cells may contribute to immune activation (20-23). TNF- $\alpha$, for example, is a protein that can exist as either a soluble molecule or a membrane-associated glycoprotein (24-26). Either form of the protein can augment B-cell expression of CD80 and other immune costimulatory molecules (27) and cause polyclonal Bcell activation (28). In this study, we examined whether the cell-free plasma of patients with SLE could also induce the expression of immune accessory molecules on human B cells.

\section{Methods}

After informed consent, blood was obtained from patients (22-69 years old) who satisfied diagnostic criteria of the American College of Rheumatology (ACR) for SLE (29) or from normal age-matched control donors. Whole blood was collected into tubes containing EDTA or heparin, and was separated immediately by centrifugation at $100 \mathrm{~g}$ at $4^{\circ} \mathrm{C}$. The plasma was harvested and stored at $-80^{\circ} \mathrm{C}$ until analyzed. Mononuclear cells were isolated from the cell pellet using densitygradient centrifugation in Histopaque 1077 (Sigma Chemical Co., St. Louis, Missouri, USA). The cells were analyzed immediately or were suspended in FCS containing 10\% DMSO for storage in liquid nitrogen. The Burkitt's lymphoma B-cell line Ramos was obtained from the American Type Culture Collection (Rockville, Maryland, USA) and cultured in RPMI-1640 supplemented with $10 \%$ FCS. Neutralization antibodies specific for human CD154 (TRAP-1), TNF- $\alpha$ (mAb1), or IFN- $\gamma$ (NIB42) were obtained from PharMingen (San Diego, California, USA). Matrix metalloproteinase inhibitors BB94 and KB-R8301 were obtained from Ko Okumura (Juntendo University, Tokyo, Japan). Sodium azide was purchased from Sigma Chemical Co.

Flow cytometry. Isolated mononuclear cells were washed and suspended in staining media (SM) consisting of RPMI-1640, $3 \%$ FCS, $0.05 \% \mathrm{NaN}_{3}$, and 1 $\mu \mathrm{g} / \mathrm{mL}$ propidium iodide (PI). The cells were stained for flow cytometry in SM containing saturating amounts of fluorochrome-conjugated mAb's. We used fluorochrome-conjugated mAb's specific for human CD54 or CD3 (Caltag Laboratories Inc., Burlingame, California, USA) or CD95 or CD154 (PharMingen). Alternatively, the cells were stained with fluorochrome-conjugated isotype control antibody (MOPC-21; PharMingen). After 30 minutes at $4^{\circ} \mathrm{C}$, the cells were washed with SM and analyzed by flow cytometry using a FACScalibur (Becton Dickinson Immunocytometry Systems, San Jose, California, USA). Dead cells that stained brightly with PI were excluded from the analyses. The relative expression of surface antigen is described as the mean fluorescence intensity ratio (MFIR). The MFIR equals the mean fluorescence intensity (MFI) of cells stained with a fluorochrome-conjugated antigen-specific $\mathrm{mAb}$ divided by the MFI of cells stained with a fluorochrome-conjugated isotype control $\mathrm{mAb}$.
Immunoprecipitation and immunoblotting of soluble CD154. Human plasma $(500 \mu \mathrm{L})$ from SLE patients or healthy donors were centrifuged at $10,000 \mathrm{~g}$ before immunoprecipitation. After preclearing with normal mouse IgG1 MOPC-21-conjugated, CNBr-activated Sepharose $(20 \mu \mathrm{L}$; Pharmacia Biotech AB, Uppsala, Sweden), aliquots were immunoprecipitated with TRAP-1-conjugated, CNBr-activated Sepharose (20 $\mu \mathrm{L})$ for 2 hours at $4^{\circ} \mathrm{C}$. After washing with wash buffer (0.05\% Tween-20 in PBS), the $1 \%$ SDS eluates were subjected to SDS-PAGE under nonreducing conditions and electroblotted onto nitrocellulose membranes (Bio-Rad Laboratories Inc., Hercules, California, USA). The blots were treated overnight at $4^{\circ} \mathrm{C}$ with blocking buffer (2\% BSA and $0.05 \%$ Tween-20 in Tris-buffered saline). The blots were washed 2 times with wash buffer (0.05\% Tween-20 in TBS) and incubated for 2 hours at room temperature with polyclonal rabbit antibody to human CD154 (C-20 or D-19; Santa Cruz Biotechnology Inc., Santa Cruz, California, USA). After 4 washings, the blots were incubated with horseradish peroxidase-conjugated (HRP-conjugated) goat anti-rabbit IgG antibody (Caltag Laboratories Inc.) followed by SuperSignal substrate (Pierce Chemical Co., Rockford, Illinois, USA). The blots were then exposed to BIOMAX x-ray film (Eastman Kodak Co., Rochester, New York, USA) for a few seconds.

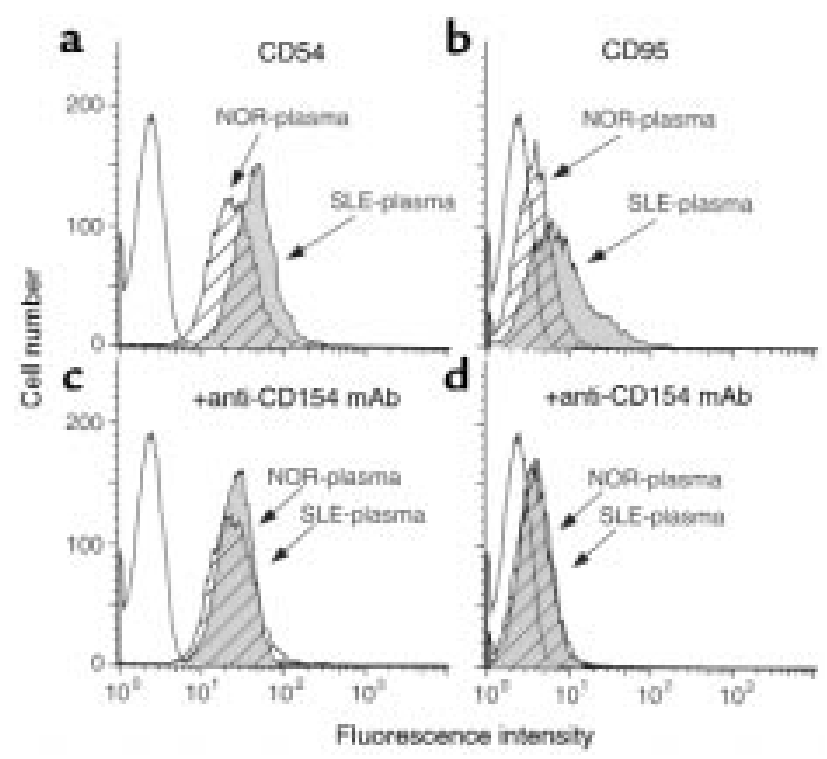

Figure 1

Changes in surface antigen phenotype of Ramos B cells after culture in human plasma depleted of fibrinogen. Ramos B cells were cultured in media supplemented with $50 \%$ human plasma either without (a and $\mathbf{b}$ ) or with (c and $\mathbf{d}$ ) a neutralizing mAb specific for CD154. The cells were examined for expression of CD54 (a and c) or CD95 (b and d) using flow cytometry. The histograms depict the relative cell number and the logarithmic fluorescence intensity. Open histograms represent staining of Ramos cells with an isotype control mAb. Hatched and filled histograms depict the staining of Ramos cells with antigenspecific $\mathrm{mAb}$ after culture in plasma from a control donor (NOR-plasma) or patient with SLE (SLE-plasma), respectively. 

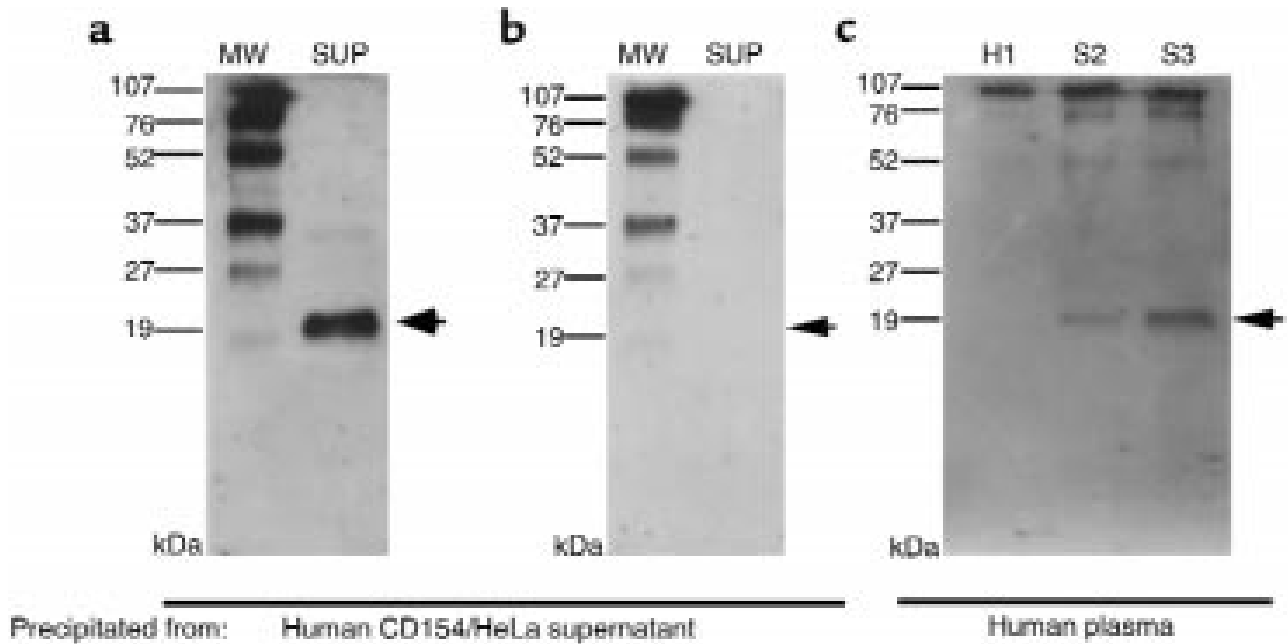

\section{Figure 2}

Immunoblot analyses of sCD154 isolated from culture supernates of human CD154-transfected HeLa cells (a and $\mathbf{b})$ or human plasma samples (c). Cell-free supernatants (SUP), or plasma from a control donor (H1) or each of 2 SLE patients (S1 and S2), were absorbed onto antiCD154 mAb-coupled Sepharose beads. The affinity-bound material was eluted, and then subjected to SDS-PAGE and immunoblot analysis with anti-CD154 antisera specific for the extracellular ( $\mathbf{a}$ and $\mathbf{c}$ ) or cytoplasmic (b) domain of CD154, respectively. The left lanes in $\mathbf{a}$ and $\mathbf{b}$ (labeled MW) are proteins of known molecular size that serve as standards. To the left of each panel are lines indicating the migration distance of each of these proteins, along with its molecular size. The arrows at the right of each panel indicate the expected migration distance of sCD154.

ELISA for soluble CD154. We measured the levels of soluble CD154 (sCD154) by an ELISA that used 2 non-cross-blocking CD154 mAb's (clones TRAP-1 and M90) obtained from Hideo Yagita and Ko Okumura (Juntendo University). For this, polystyrene microtiter plates (Corning-Costar Corp., Cambridge, Massachusetts, USA) were coated with TRAP- 1 at $3 \mu \mathrm{g} / \mathrm{mL}$ in 0.1 $\mathrm{M}$ phosphate buffer ( $\mathrm{pH}$ 9.0). The plates were washed with PBS and treated for 2 hours at room temperature with blocking buffer (10\% FCS and $0.01 \%$ sodium azide in PBS). The plates were washed 4 times with wash buffer $(0.05 \%$ Tween-20 in PBS), and then incubated overnight at $4{ }^{\circ} \mathrm{C}$ with human plasma diluted in blocking buffer. For our standard curve, we used a recombinant sCD154 obtained from Bender MedSystems (Vienna, Austria). The plates were washed 4 times with wash buffer, and then incubated for 1 hour with biotinylated $\mathrm{M} 90 \mathrm{mAb}$ at $3 \mu \mathrm{g} / \mathrm{mL}$ in blocking buffer. Washed plates were then treated sequentially with avidin and then biotinylated HRP (biotin-HRP) (Elite VECTASTAIN; Vector Laboratories, Burlingame, California, USA). After washing plates with blocking buffer, the plates were allowed to react with its substrate, 3,3',5,5'-tetramethylbenzidine (TMB) peroxidase (Kirkegaard \& Perry Laboratories Inc., Gaithersburg, Maryland, USA). The OD at $450 \mathrm{~nm}$ was determined using an ELISA microplate reader (Molecular Devices Corp., Sunnyvale, California, USA).

Quantitative CD154 RT-PCR ELISA. ELISA-based quantitative competitive RT-PCR for CD154 was as described previously (30). Total RNA from PBMCs (1-5 $\times 10^{6}$ cells) was extracted with the Total RNeasy Kit (QIAGEN Inc., Valencia, California, USA). The competitor RNA was generated from the insert-containing
CD154 cDNA cloned into pBluescript (Stratagene, La Jolla, California, USA), using the Superscript Preamplification System. Varying amounts of competitor RNA were added to separate wells of isolated total RNA that subsequently were converted into cDNA using the First Strand cDNA Synthesis Kit (GIBCO BRL, Grand Island, New York, USA). PCR reactions were performed for 35 cycles, and the products were purified with a QIAquick Spin PCR Purification Kit (QIAGEN Inc.). Standard DNA of CD154 was amplified from pBSII KS using T3 primer (5'-ATTAACCCTCACTAAAG-3') and T7 primer (5'-biotin-AATACGACTCACTATAG-3'). PCR product serial dilutions of $1: 3$, ranging from $1 \mathrm{pM}$ to $500 \mathrm{aM}$, were used to generate a standard curve.

The biotinylated PCR products were captured onto microtiter plates (Becton Dickinson Labware, Franklin Lakes, New Jersey, USA) coated with streptavidin (Sigma Chemical Co.). PCR products were loaded into 2 wells and incubated at room temperature for 1 hour. The plates were treated with $0.1 \mathrm{~N} \mathrm{NaOH}$ for 10 minutes to remove the sense-strand DNA, and then were washed with $0.1 \times$ SSC and BW buffer. The plates were incubated with oligonucleotide probes labeled with digoxigenin-11-dideoxy-UTP (Boehringer Mannheim, Indianapolis, Indiana, USA) at $65^{\circ} \mathrm{C}$ for 20 minutes and then at $42^{\circ} \mathrm{C}$ for 90 minutes. After washing with HYBE buffer ( $6 \times$ SSC, $0.1 \%$ N-lauroyl sarlosine) and blocking buffer $(0.5 \%$ Genius blocking reagent [Boehringer Mannheim] in Tris-HCl buffer), HRP-conjugated antidigoxigenin antibody (Boehringer Mannheim) in blocking buffer was added and incubated for 30 minutes at room temperature; then plates were allowed to react with its substrate TMB peroxidase. The OD at $450 \mathrm{~nm}$ was determined using an ELISA microplate reader. 

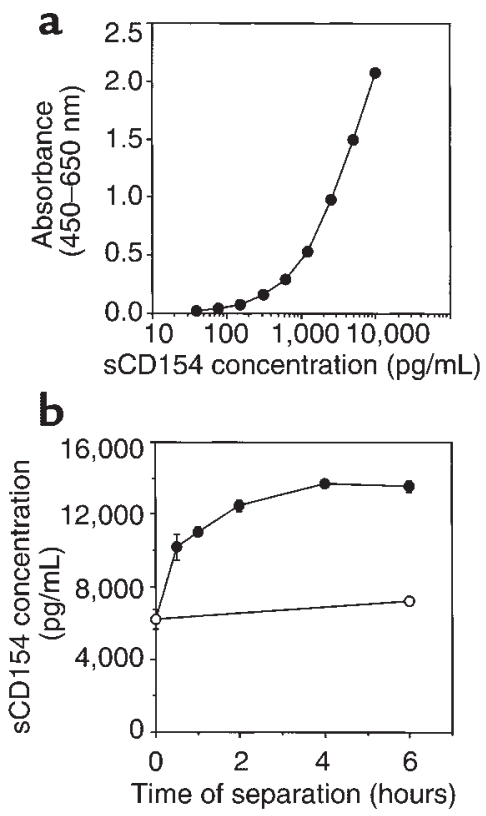

c

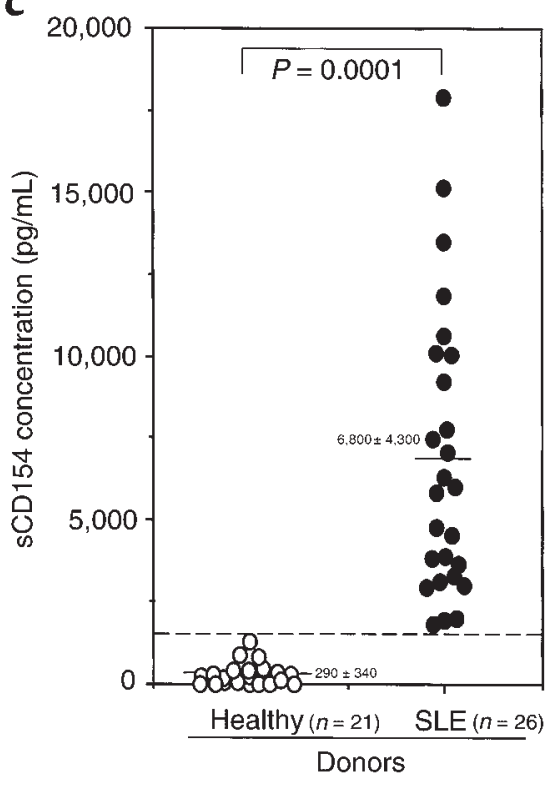

Figure 3

ELISA to detect sCD154 concentrations in human plasma. (a) A standard curve for serial dilutions of a recombinant sCD154 placed into separate wells of a microtiter plate precoated with anti-CD154 mAb (TRAP1). Bound recombinant sCD154 was detected using a non-crossreacting, biotinylated anti-CD154 mAb (M90). The mean absorbance of triplicate wells is indicated on the ordinate, and the logarithmic concentration (picograms per milliliter) of the recombinant sCD154 is provided on the abscissa. (b) Indicated are the measured concentrations of sCD154 in plasma isolated at various times after phlebotomy from whole blood kept at room temperature (filled circles) or at $4^{\circ} \mathrm{C}$ (open circles). The calculated concentration of sCD154 is listed on the ordinate (picograms per milliliter), and the time after phlebotomy prior to plasma separation is listed in hours on the abscissa. Each point represents the mean value of triplicate wells, and the error bars indicate the SE. (c) The concentrations of sCD154 (picograms per milliliter) that were detected in the plasma of each of 21 control donors (open circles) or 26 patients with SLE (filled circles) are indicated. Each point represents the concentration found in a single donor. The bar indicates the mean concentration for the healthy and SLE groups. The numbers indicate the mean concentration for each group \pm SD about the mean. A dotted line represents the mean +3 SD of sCD154 in control donors. The $P$ value is calculated for the difference between the means of the 2 groups $(P<0.0001)$.

Standard curves plotting the moles of RNA product versus OD were made for the standard cDNA reactions. The equations describing these standard curves were then used to calculate the moles of wild-type or competitor DNA present in the unknown PCR reactions, based on the ODs obtained in the ELISA readings. The ratio of wild-type DNA to competitor DNA was then plotted against the known quantity of competitor RNA added in the initial samples. The ratio of 1 was interpreted to mean that the amount of target RNA equaled the amount of competitor RNA added to the sample. The molecules of target RNA per PBMC were then calculated based on the following formula: [(moles target CD154 RNA $) \times(6 \times$ $10^{23}$ molecules per mole) $\times$ (dilution factor of test RNA)]/(percent of PBMCs in total cell population).

Measurement of disease activity. The SLE disease activity index (SLEDAI) score and anti-dsDNA Ab titer were determined at the time of blood sampling. Disease activity for lupus patients was measured using the SLEDAI score (31). Plasma anti-dsDNA levels were measured using the Farr technique at Specialty Laboratories (Santa Monica, California, USA).

Statistical analyses. The mean sCD154 plasma concentrations of patients with SLE and the mean concentrations of control donors were compared using the
Mann-Whitney nonpaired nonparametric test. Relationships between the level of sCD154 protein and CD154 mRNA or anti-dsDNA Ab titer in SLE patients were both analyzed with the Pearson correlation test. Because the Pearson correlation test assumes the usual bivariate Gaussian distribution, we also analyzed these relationships using the Spearman rank order correlation test, which makes less stringent assumptions.

\section{Results}

Plasma from patients with active SLE can upregulate expression of immune accessory molecules on Ramos B cells. We assessed phenotypic changes in Ramos B cells after culturing the cells in media supplemented with plasma from patients with SLE. Briefly, Ramos B cells were cultured for 48 hours in media supplemented with 50\% plasma from patients with SLE or from normal control donors. We found that the plasma from patients with active SLE could induce such cells to express higher levels of CD54 and CD95, whereas plasma from healthy donors could not (Figure 1, a and b). Moreover, plasma from patients with active SLE could induce Ramos B cells to express CD80 after 72 hours in culture (data not shown). The ability of SLE plasma to induce increased expression of CD54, CD95, or CD80 could be 
inhibited by anti-human CD154 mAb (Figure 1, $\mathrm{c}$ and d), but not by anti-human TNF- $\alpha$ or anti-human IFN$\gamma$ mAb's (data not shown). The increased expression of CD54, CD80, or CD95 is similar to that noted for leukemia $\mathrm{B}$ cells stimulated with cells expressing membrane-bound CD154 (32). Collectively, these data imply that the plasma of patients with active SLE could induce expression of immune accessory molecules on $\mathrm{B}$ cells, and that this activity was mediated by SCD 154 . sCD154 in plasma of patients with active SLE. To look for sCD154 in SLE plasma, we first established whether we could detect sCD154 in the culture supernatants of a HeLa cell line (designated CD154-HeLa) that was transfected with an expression vector encoding the full-length human CD154. Culture supernatants of CD154-HeLa were incubated with Sepharose beads conjugated with anti-CD154 mAb. This process captured a protein of approximately 20 $\mathrm{kDa}$ that could be identified with immunoblot analysis using an antibody specific for the $\mathrm{COOH}$-terminal (extracellular) region of CD154 (Figure 2a), but not with an antibody specific for the $\mathrm{NH}_{2}$-terminal (cytoplasmic) region of CD154 (Figure $2 \mathrm{~b}$ ). The size of this protein was similar to the 19 - to $20-\mathrm{kDa}$ sCD154 glycoprotein isolated from the supernatants of activated $\mathrm{T}$ cells (33). We then examined whether we could immunoprecipitate sCD154 from the plasma of patients with active SLE or healthy donors. As seen in Figure 2c, sCD154 could be isolated from the plasma of patients with active SLE (S1 and S2), but not from plasma of a normal healthy donor (H1).

To measure the levels of SCD154 in the plasma of SLE patients, we developed a sensitive and specific sandwich ELISA using 2 non-cross-blocking anti-CD154 mAb's (clones TRAP-1 and M90). Recombinant homotrimeric SCD154 was used as a positive standard control for the ELISA (Figure 3a). The minimum concentration of sCD154 that we could detect with this assay was approximately $40 \mathrm{pg} / \mathrm{mL}$. The specificity of this ELISA was confirmed by its ability to detect human sCD154 protein, but not murine sCD154, human soluble Fas ligand, or human soluble CD27 (data not shown).

To assess whether the $\mathrm{SCD} 154$ detected in the plasma of patients with SLE was released from cells after phlebotomy, we examined changes in plasma levels of sCD154 from collected blood samples over time. Plasma was isolated at various times after phlebotomy from whole-blood samples kept at room temperature or at $4^{\circ} \mathrm{C}$. The measured sCD154 levels in blood samples kept at $4^{\circ} \mathrm{C}$ were constant over the first 6 hours after sample collection (Figure $3 \mathrm{~b}$ ). Moreover, these levels were similar to those found in plasma processed immediately after phlebotomy. However, the sCD154 levels in plasma isolated from whole blood kept at room temperature increased over time, reaching a plateau value after 2 hours (Figure $3 \mathrm{~b}$ ). Addition of matrix metalloproteinase inhibitors BB94 or KBR8301 to the blood samples immediately after phlebotomy could inhibit this rise in $\mathrm{sCD} 154$ over time
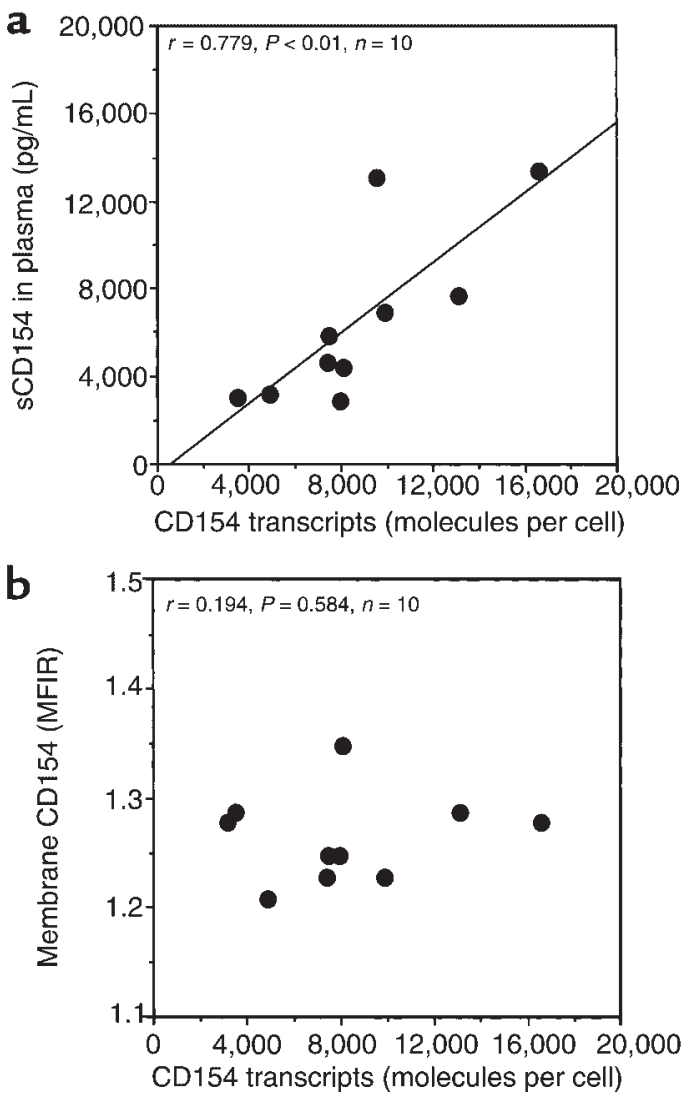

Figure 4

Comparison of the numbers of CD154 transcripts per cell with the measured sCD154 plasma concentration or the intensity of CD154 surface membrane expression on blood mononuclear cells of patients with SLE. The numbers of CD154 transcripts per freshly isolated mononuclear cells of 10 patients with SLE are indicated on the abscissa. (a) Each dot represents the mean plasma concentration of SCD154 detected for each patient, as indicated on the ordinate (picogram per milliliter). These points are plotted against the numbers of CD154 transcripts per cell detected in the mononuclear cells isolated from the whole-blood sample from which each plasma sample was derived. The line depicts the best-fit correlation between the levels of CD154 transcripts per cell and the plasma concentration of SCD154 (Pearson $r=0.779, P=0.01$ ). (b) Each dot represents the intensity of CD154 membrane expression on the blood mononuclear cells of each patient, indicated on the ordinate as the MFIR of cells stained with anti-CD154 mAb and a control $\mathrm{mAb}$ of the same isotype. The line depicts the best-fit correlation between the levels of CD154 transcripts per cell and the intensity of CD154 surface membrane expression (Spearman $r=0.194, P=0.584$ ).

(data not shown). However, addition of sodium azide to blood samples in order to reach a final concentration of $0.05 \%$ did not inhibit the release of sCD 154 over time (data not shown). These data indicate that sCD154 may be cleaved from membrane CD154 by a matrix metalloproteinase and released into the plasma ex vivo through a process that does not require oxidative phosphorylation. Nevertheless, patients with active SLE apparently have elevated levels of SCD154 in vivo, because high levels of sCD154 could be detected in the plasma from such patients even when it was isolated immediately after phlebotomy. 


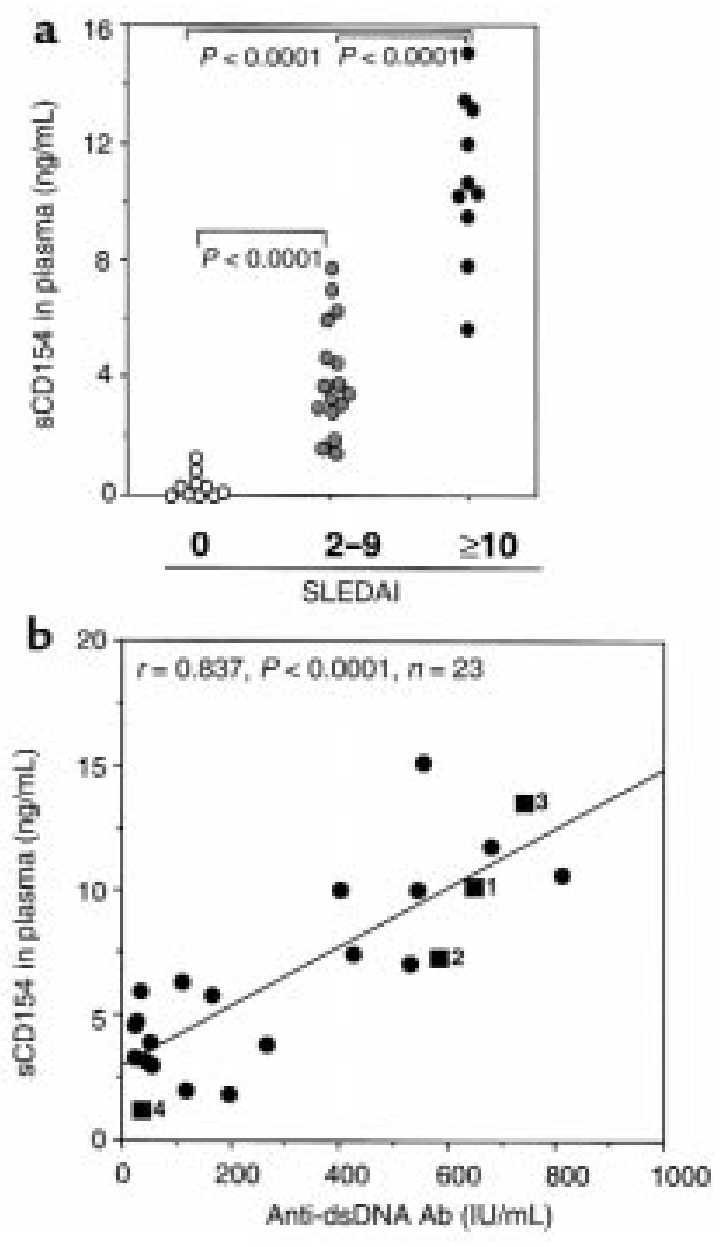

\section{Figure 5}

Relationship between sCD154 plasma concentration and SLE disease activity. (a) Described are the measured sCD154 plasma concentrations of control donors (open circles in column 0), SLE patients with low disease activity (SLEDAI between 2 and 9; shaded circles in column labeled 2-9), and patients with active SLE (SLEDAI $\geq 10$; filled circles in the right column labeled $\geq 10$ ), as indicated on the abscissa (nanogram per milliliter). Each dot represents the measured plasma concentration for an individual subject. The $P$ values, indicating the differences between the mean values for each group, are indicated at the top of the figure. The difference between the inactive and active SLE groups is statistically significant $(P<0.0001)$. (b) Described are the relationships between measured plasma concentrations of sCD154 (as indicated on the ordinate in nanogram per milliliter) and the anti-dsDNA Ab levels in each of 23 SLE patient samples, as indicated on the abscissa $(I U / \mathrm{mL})$. The line represents the best-fit correlation between the plasma concentrations of sCD154 and the anti-dsDNA Ab levels (Spearman $r=0.758, P<$ 0.001 ). Four of the values represent measurements made of serial samples obtained from the same patient (filled squares labeled 1, 2, 3 , and 4). Square 1 is the initial sample taken before immune suppressive therapy. Samples for squares 2, 3, and 4 were obtained from the same patient 4,8 , and 17 weeks later, respectively.

We determined the levels of sCD154 in the plasma of 21 healthy subjects and 26 SLE patients who had different degrees of disease activity. Plasma samples were isolated from whole-blood samples that were chilled immediately to $4^{\circ} \mathrm{C}$ after phlebotomy and then processed within 30 minutes (Figure 3c). In the control group, the plasma concentration of sCD154 was $290 \pm$ $340 \mathrm{pg} / \mathrm{mL}$ (mean $\pm \mathrm{SD}$ ). Moreover, half of the tested plasma samples from the control donors did not have detectable amounts of sCD154 $(<40 \mathrm{pg} / \mathrm{mL})$, as determined by ELISA (Figure 3c). However, the plasma of SLE patients had significantly higher levels of sCD154 $(6,800 \pm 4,300 \mathrm{pg} / \mathrm{mL}$; mean $\pm \mathrm{SD})$ than the control plasma samples $(P<0.0001$ by Mann-Whitney unpaired nonparametric test).

Correlation between expression levels of CD154 mRNA and $s C D 154$. To examine the levels of CD154 mRNA in the blood mononuclear cells of patients with SLE, we used an ELISA-based quantitative competitive RTPCR to measure the numbers of CD154 transcript per cell, as described (30). This method titers known amounts of a CD 154 competitor transcript containing an engineered mutation that can be detected with oligonucleotide hybridization.

We found that the blood mononuclear cells of 10 patients with active SLE had an average of 7,800 $\pm 1,100$ (SD) transcripts of CD154 mRNA per cell. The number of transcripts per cell was significantly higher than that noted in the blood mononuclear cells of 10 healthy control donors $(P<0.001$ by Mann-Whitney unpaired nonparametric test). Furthermore, we found that the levels of CD154 transcripts per cell for individual patients correlated with the relative levels of SCD154 detected in their respective plasma samples (Pearson $r$ $=0.779, P=0.008$; Spearman $r=0.782, P<0.02$ ) (Figure 4a). However, the relative levels of sCD154 transcripts did not have such a significant correlation with the relative MFIR values for membrane-bound CD154 detected on the cells in respective samples using flow cytometry (Spearman $r=0.194, P=0.584$ ) (Figure $4 \mathrm{~b}$ ).

Correlation between $s C D 154$ and dsDNA titer. We compared the SLEDAI of each patient with the level of sCD154 detected in the plasma. The levels of sCD154 were highest in the plasma of patients with highly active disease who had a SLEDAI score of 10 or greater. These 10 patients had higher mean plasma levels of sCD154 (mean \pm SD of $10,680 \pm 2,840 \mathrm{pg} / \mathrm{mL}$ ). Patients with lessactive disease (SLEDAI scores between 2 and 9) had a significantly lower mean plasma level of sCD154 $(m=3,880 \pm 2,080 \mathrm{pg} / \mathrm{mL}, n=16)$ than patients with SLEDAI scores of 10 or greater $(P<0.0001$ by MannWhitney unpaired nonparametric test; Figure $5 \mathrm{a}$ ). Nevertheless, the mean sCD154 plasma level of these patients was still significantly higher than that of normal donors $(m=423 \pm 437 \mathrm{pg} / \mathrm{mL}, n=10, P<0.0001$ by Mann-Whitney unpaired nonparametric test; Figure 5a).

We also compared the anti-dsDNA Ab titers with sCD154 concentration in SLE plasma. A highly significant linear correlation was found between plasma antidsDNA Ab titers and sCD154 plasma levels (Pearson $r$ $=0.837, P<0.0001$; Spearman $r=0.758, P<0.001$ $[n=23]$ ) (Figure 5b). We also examined serial plasma samples collected over a 17 -week period from one patient who initially had a high plasma level of sCD154 
$(10,194 \mathrm{pg} / \mathrm{mL})$ and a high titer of anti-dsDNA Ab (650 $\mathrm{IU} / \mathrm{mL}$ ) (Figure $5 \mathrm{~b}$, square 1 ). This patient's plasma levels of sCD154 and titer of anti-dsDNA Ab rose (Figure $5 b$, square 2 ) and fell (Figure 5b, squares 3 and 4 ) concomitantly after treatment, to final values of 1,114 $\mathrm{pg} / \mathrm{mL}$ and $34 \mathrm{IU} / \mathrm{mL}$, respectively. However, no correlation was found between sCD154 plasma levels and proteinuria (data not shown).

\section{Discussion}

In this study, we found that cell-free plasma of patients with SLE could induce Ramos B cells to express high levels of CD54 and CD95. The ability of SLE plasma samples to induce such phenotypic changes could be abrogated by pretreating the plasma with neutralizing mAb's specific for CD154 (Figure 1). Immunoprecipitation and immunoblot analyses revealed that the plasma from patients with active disease possessed a protein of approximately $20 \mathrm{kDa}$ that reacted with mAb's specific for the extracellular domain of CD154 (Figure 2 ). Collectively, these data indicate that the plasma of patients with active SLE contains a CD154 polypeptide that has biologic activity.

CD154 exists ordinarily as a $39-\mathrm{kDa}$ type II membrane glycoprotein (34). This protein is expressed transiently by activated CD4+ $\mathrm{T}$ cells $(30,35-37)$. However, CD154 also can be cleaved from the cell surface of activated $\mathrm{T}$ cells, releasing an sCD154 of approximately 18-20 kDa (38). This sCD154 can apparently form homotrimers that can induce B-cell activation or differentiation $(33,38)$. Moreover, several in vitro studies have revealed that $\mathrm{sCD} 154$, or a soluble recombinant CD154 chimeric protein, can induce antigen-presenting cells (APCs) to express immune accessory molecules in a manner similar to that of plasma from patients with active $\operatorname{SLE}(33,39,40)$.

The sCD154 in plasma of patients with active SLE is most likely derived from the membrane-bound form of CD154 on activated T cells. Previous reports demonstrated that the absolute numbers of blood T cells that express surface CD154 are increased in patients with active lupus $(16,17)$. Moreover, $\mathrm{T}$ cells from SLE patients could also maintain high-level cell-surface expression of CD154 in culture, indicating that such $\mathrm{T}$ cells have constitutive or exaggerated expression of CD154. Consistent with this, we found that the blood mononuclear cells of patients with active SLE expressed significantly higher levels of CD154 transcripts per cell than the blood mononuclear cells of control donors. However, the expression levels of surface CD154 on such cells did not correlate well with disease activity (data not shown). On the other hand, the plasma concentrations of sCD154 did have a significant correlation with disease activity (Figure 5). The soluble molecule does not appear to be encoded by a truncated CD154 mRNA, because only full-length transcripts could be identified in the mononuclear cells of patients with SLE (data not shown). Furthermore, the level of sCD154 found in the plasma had a signifi- cant correlation with the level of CD154 mRNA detected in blood mononuclear cells using quantitative RTPCR (Figure 4a). As such, it appears that SCD154 in the plasma of patients with SLE results from cleavage of the full-length form of the CD154 molecule.

Consistent with this notion, we found that the plasma concentrations of sCD154 increased over time in blood samples that were not refrigerated (Figure $3 \mathrm{~b}$ ). The ex vivo increase in the plasma concentrations of SCD154 could be inhibited by chilling the sample to $4^{\circ} \mathrm{C}$ or by adding matrix metalloproteinase inhibitors, such as BB94 or KB-R8301, to the collected sample. This inhibition indicates that the release of sCD154 is a temperature-dependent process that may require a matrix metalloproteinase. This process is similar to that required for release of TNF- $\alpha$ or soluble Fas ligand $(41,42)$.

Despite the ex vivo production of SCD154, we found that plasma immediately isolated from the collected blood samples of patients with SLE still contained high concentrations of sCD154. This concentration does not appear secondary to the immediate release of sCD154. One study indicated that platelets can express CD154 within seconds after activation in vitro (43). For this reason, we collected the blood samples in tubes containing heparin sulfate to prevent thrombus formation and platelet activation. Because we found that the ex vivo release of sCD154 was temperature-dependent, we chilled subsequent blood samples immediately to $4^{\circ} \mathrm{C}$ and processed them soon thereafter. Blood samples were collected from SLE patients and control donors in an identical fashion. If our methods stimulated an immediate release of sCD154 from platelets, then the plasma collected from control donors would also be expected to contain elevated levels of sCD154. Instead, the plasma from patients with active SLE had consistently higher levels of sCD154 than plasma from control donors. Furthermore, the significant correlation between the levels of lymphocyte CD154 mRNA and plasma concentrations of sCD154 (Figure 4a) also argues against the notion that sCD154 is released from platelets at the time of sample collection or processing. Instead, it appears that patients with active SLE have elevated levels of sCD154 in vivo.

The elevated plasma levels of sCD154 appear to be associated with autoimmune disease activity. The plasma samples from most normal donors did not have sCD154 at levels that could be detected in our assay (e.g., $\geq 40 \mathrm{pg} / \mathrm{mL}$ ). Moreover, the plasma samples of patients with other diseases, such as progressive systemic sclerosis, pneumonia, Sjögren's syndrome, or multiple myeloma, also had little or no detectable sCD154 (data not shown). On the other hand, all the plasma samples from patients with SLE contained detectable amounts of sCD154. Furthermore, the sCD154 levels in plasma correlated with anti-dsDNA $A b$ titers and clinical disease activity, as assessed by the SLEDI (31) (Figure 5).

In view of this data, we hypothesize that the correlation defines a causal relationship. Similar to membrane-bound CD154, elevated expression of sCD154 
may contribute to immune activation of APCs and stimulation of autoantibody-producing B cells in patients with SLE. In addition to its ability to activate $\mathrm{B}$ cells, monocytes, or dendritic cells, CD154 also may induce inflammatory changes in CD40-bearing nonhematopoietic cells. For example, endothelial cells can be induced to upregulate surface expression of CD54, CD62E, and CD106 (44) upon CD40 ligation, thereby assuming a phenotype more conducive to inflammation. Conceivably, the vasculitis that is often observed in patients with highly active SLE may be due, in part, to high levels of circulating sCD154. Consistent with this notion, we found that patients with active vasculitis in the setting of rheumatoid arthritis also have high plasma levels of sCD154 (data not shown; T. Tamura, S. Kobayashi, K. Kato, T.J. Kipps, and H. Brando, manuscript in preparation).

Similarly, it is possible that sCD154 may play a role in renal pathology that often develops in patients with SLE. Because monomeric, and even homotrimeric, sCD154 falls below the 64-kDa cutoff for renal filtration, sCD154 may pass through the renal glomerulus and become sequestered or undergo absorption in the proximal tubules. Accordingly, sCD154 may have a greater influence on proximal tubular epithelial cells than the blood lymphocytes that express this molecule on the cell surface. It is noteworthy that proximal tubular epithelial cells also express CD40. Moreover, ligation of CD40 on these cells stimulates them to express inflammatory chemokines, such as IL-8, MCP-1, and RANTES (45). Although we failed to detect a significant correlation between the plasma level of sCD154 and proteinuria in patients with SLE, further studies are necessary to determine whether the plasma levels of sCD154 correlate with progression of lupus nephropathy.

Future studies are required to determine whether the plasma level of sCD154 has value in predicting flare-ups in SLE disease activity. It is possible that the level of sCD154 may rise and fall faster than that of anti-dsDNA $\mathrm{Ab}$ 's during flare-ups and remissions in disease activity, respectively. If so, then assessing the plasma concentration of sCD154 may prove to be a more reliable way to monitor disease activity in patients with SLE.

\section{Acknowledgments}

We are grateful to Hideo Yagita and Ko Okumura for providing us with antibodies to human CD154. We also thank Manami Seki for technical assistance. This work was supported in part by P50-AR44850 from the National Institutes of Health.

1. Swaak, A.J., Aarden, L.A., Statius van Eps, L.W., and Feltkamp, T.E. 1979. Anti-dsDNA and complement profiles as prognostic guides in systemic lupus erythematosus. Arthritis Rheum. 22:226-235.

2. Takasaki, Y., et al. 1998. Expression of costimulatory molecule CD80 on peripheral blood T cells in patients with systemic lupus erythematosus. J. Rheumatol. 25:1085-1091.

3. Kovacs, B., Thomas, D.E., and Tsokos, G.C. 1996. Elevated in vivo expression of the costimulatory molecule B7-BB1 (CD80) on antigen presenting cells from a patient with SLE. Clin. Exp. Rheumatol. 14:695-697.
4. Folzenlogen, D., Hofer, M.F., Leung, D.Y., Freed, J.H., and Newell, M.K. 1997. Analysis of CD80 and CD86 expression on peripheral blood B lymphocytes reveals increased expression of CD86 in lupus patients [see comments]. Clin. Immunol. Immunopathol. 83:199-204.

5. Mysler, E., et al. 1994. The apoptosis-1/Fas protein in human systemic lupus erythematosus. J. Clin. Invest. 93:1029-1034.

6. Finck, B.K., Linsley, P.S., and Wofsy, D. 1994. Treatment of murine lupus with CTLA4Ig. Science. 265:1225-1227.

7. Kaneko, Y., et al. 1996. CD40-mediated stimulation of B1 and B2 cells: implication in autoantibody production in murine lupus. Eur. J. Immunol. 26:3061-3065.

8. Linsley, P.S., et al. 1991. CTLA-4 is a second receptor for the B cell activation antigen B7. J. Exp. Med. 174:561-569.

9. Noelle, R.J., et al. 1992. A 39-kDa protein on activated helper T cells binds CD40 and transduces the signal for cognate activation of B cells. Proc. Natl. Acad. Sci. USA. 89:6550-6554.

10. Ranheim, E.A., and Kipps, T.J. 1993. Activated T cells induce expression of B7/BB1 on normal or leukemic B cells through a CD40-dependent signal. J. Exp. Med. 177:925-935.

11. Banchereau, J., et al. 1994. The CD40 antigen and its ligand. Annu. Rev. Immunol. 12:881-922.

12. Clark, E.A., and Ledbetter, J.A. 1994. How B and T cells talk to each other. Nature. 367:425-428.

13. Durie, F.H., Foy, T.M., Masters, S.R., Laman, J.D., and Noelle, R.J. 1994. The role of CD40 in the regulation of humoral and cell-mediated immunity. Immunol. Today. 15:406-411.

14. Yang, Y., and Wilson, J.M. 1996. CD40 ligand-dependent T cell activation: requirement of B7-CD28 signaling through CD40. Science. 273:1862-1864

15. Grewal, I.S., et al. 1996. Requirement for CD40 ligand in costimulation induction, T cell activation, and experimental allergic encephalomyelitis. Science. 273:1864-1867.

16. Koshy, M., Berger, D., and Crow, M.K. 1996. Increased expression of CD40 ligand on systemic lupus erythematosus lymphocytes. J. Clin. Invest. 98:826-837.

17. Desai-Mehta, A., Lu, L., Ramsey-Goldman, R., and Datta, S.K. 1996. Hyperexpression of CD40 ligand by B and T cells in human lupus and its role in pathogenic autoantibody production. J. Clin. Invest. 97:2063-2073.

18. Early, G.S., Zhao, W., and Burns, C.M. 1996. Anti-CD40 ligand antibody treatment prevents the development of lupus-like nephritis in a subset of New Zealand black x New Zealand white mice. Response correlates with the absence of an anti-antibody response. J. Immunol. 157:3159-3164.

19. Ma, J., et al. 1996. Autoimmune lpr/lpr mice deficient in CD40 ligand: spontaneous Ig class switching with dichotomy of autoantibody responses. J. Immunol. 157:417-426.

20. Swaak, A.J., Hintzen, R.Q., Huysen, V., van den Brink, H.G., and Smeenk, J.T. 1995. Serum levels of soluble forms of T cell activation antigens CD27 and CD25 in systemic lupus erythematosus in relation with lymphocytes count and disease course. Clin. Rheumatol. 14:293-300.

21. Font, J., et al. 1996. Elevated soluble CD27 levels in serum of patients with systemic lupus erythematosus. Clin. Immunol. Immunopathol. 81:239-243.

22. Jodo, S., et al. 1997. Serum levels of soluble Fas/APO-1 (CD95) and its molecular structure in patients with systemic lupus erythematosus (SLE) and other autoimmune diseases. Clin. Exp. Immunol. 107:89-95.

23. Gattorno, M., et al. 1998. Differences in tumor necrosis factor-alpha soluble receptor serum concentrations between patients with HenochSchönlein purpura and pediatric systemic lupus erythematosus: pathogenetic implications. J. Rheumatol. 25:361-365.

24. Kriegler, M., Perez, C., DeFay, K., Albert, I., and Lu, S.D. 1988. A novel form of TNF/cachectin is a cell surface cytotoxic transmembrane protein: ramifications for the complex physiology of TNF. Cell. 53:45-53.

25. Luettig, B., Decker, T., and Lohmann-Matthes, M.L. 1989. Evidence for the existence of two forms of membrane tumor necrosis factor: an integral protein and a molecule attached to its receptor. J. Immunol. 143:4034-4038.

26. Grell, M., et al. 1995. The transmembrane form of tumor necrosis factor is the prime activating ligand of the $80 \mathrm{kDa}$ tumor necrosis factor receptor. Cell. 83:793-802.

27. Ranheim, E.A., and Kipps, T.J. 1995. Tumor necrosis factor-alpha facilitates induction of CD80 (B7-1) and CD54 on human B cells by activated T cells: complex regulation by IL-4, IL-10, and CD40L. Cell. Immunol. 161:226-235.

28. Macchia, D., et al. 1993. Membrane tumour necrosis factor-alpha is involved in the polyclonal B-cell activation induced by HIV-infected human T cells. Nature. 363:464-466.

29. Tan, E.M., et al. 1982. The 1982 revised criteria for the classification of systemic lupus erythematosus. Arthritis Rheum. 25:1271-1277.

30. Cantwell, M.J., Hua, T., Pappas, J., and Kipps, T.J. 1997. Acquired CD40ligand deficiency in chronic lymphocytic leukemia. Nat. Med. 3:984-989. 31. Liang, M.H., Socher, S.A., Larson, M.G., and Schur, P.H. 1989. Reliabili- 
ty and validity of six systems for the clinical assessment of disease activity in systemic lupus erythematosus. Arthritis Rheum. 32:1107-1118

32. Kato, K., Cantwell, M.J., Sharma, S., and Kipps, T.J. 1998. Gene transfer of CD40-ligand induces autologous immune recognition of chronic lymphocytic leukemia B cells. J. Clin. Invest. 101:1133-1141.

33. Mazzei, G.J., et al. 1995. Recombinant soluble trimeric CD40 ligand is biologically active. J. Biol. Chem. 270:7025-7028.

34. Armitage, R.J., et al. 1992. Molecular and biological characterization of a murine ligand for CD40. Nature. 357:80-82.

35. Roy, M., Waldschmidt, T., Aruffo, A., Ledbetter, J.A., and Noelle, R.J 1993. The regulation of the expression of $g \mathrm{p} 39$, the CD40 ligand, on normal and cloned CD4+ T cells. J. Immunol. 151:2497-2510.

36. Castle, B.E., Kishimoto, K., Stearns, C., Brown, M.L., and Kehry, M.R. 1993. Regulation of expression of the ligand for CD40 on T helper lymphocytes. J. Immunol. 151:1777-1788.

37. Hermann, P., et al. 1993. Expression of a 32-kDa ligand for the CD40 antigen on activated human T lymphocytes. Eur. J. Immunol. 23:961-964. 38. Graf, D., et al. 1995. A soluble form of TRAP (CD40 ligand) is rapidly released after T cell activation. Eur. J. Immunol. 25:1749-1754

39. Wiley, J.A., Geha, R., and Harmsen, A.G. 1997. Exogenous CD40 ligand induces a pulmonary inflammation response. J. Immunol. 158:2932-2938.

40. Jumper, M.D., Nishioka, Y., Davis, L.S., Lipsky, P.E., and Meek, K. 1995. Regulation of human B cell function by recombinant CD40 ligand and other TNF-related ligands. J. Immunol. 155:2369-2378.

41. Crowe, P.D., et al. 1995. A metalloprotease inhibitor blocks shedding of the $80-\mathrm{kD}$ TNF receptor and TNF processing in T lymphocytes. J. Exp. Med. 181:1205-1210.

42. Kayagaki, N., et al. 1995. Metalloproteinase-mediated release of human Fas ligand. J. Exp. Med. 182:1777-1783.

43. Henn, V., et al. 1998. CD40 ligand on activated platelets triggers an inflammatory reaction of endothelial cells. Nature. 391:591-594.

44. Yellin, M.J., et al. 1995. Functional interactions of T cells with endothelial cells: the role of CD40L-CD40-mediated signals. J. Exp. Med. 182:1857-1864.

45. van Kooten, C., et al. 1997. Possible role for CD40-CD40L in the regulation of interstitial infiltration in the kidney. Kidney Int. 51:711-721. 